\title{
Negative Conditional Entropy of Post-Selected States
}

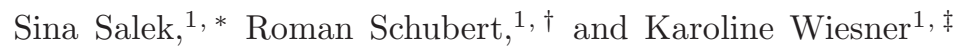 \\ ${ }^{1}$ School of Mathematics \\ The University of Bristol, Bristol BS8 1TW, United Kingdom
}

\begin{abstract}
We define an quantum entropy conditioned on post-selection which has the von Neumann entropy of pure states as a special case. This conditional entropy can take negative values which is consistent with part of a quantum system containing less information than the whole which can be in a pure state. The definition is based on generalised density operators for post-selected ensembles. The corresponding density operators are consistent with the quantum generalisation of classical conditional probabilities following Dirac's formalism of quasi-probability distributions.
\end{abstract}

PACS numbers: 03.65.Ta, 03.65.Wj, 03.65.Ca, 03.67.-a

\section{INTRODUCTION}

Post-selection refers to keeping the record of the outcome of some ensemble quantum measurement only for those parts of the ensemble which at a later point in time are in a desired, so-called postselected state and discarding the remaining results. Ensembles prepared in a state $|\psi\rangle$ and post-selected in a state $|\phi\rangle$ are described by a generalised density operator as [1, 2],

$$
\rho_{\psi \mid \phi}:=\frac{|\psi\rangle\langle\phi|}{\langle\phi \mid \psi\rangle}
$$

This generalisation is appropriate when a weak or no measurement has been performed between pre- and postselection. For a proposal that incorporates strong measurements see [3]. Nevertheless, we restrict our attention to the case where no strong measurement has been performed. These generalised density operators for postselected ensembles are used to obtain the so-called weak values $\Pi_{w}$ [4] of an operator $\Pi$ as

$$
\Pi_{w}=\operatorname{Tr}\left[\rho_{\psi \mid \phi} \Pi\right] .
$$

Experimentally, a weak value is obtained by weakly coupling an ensemble of states to a measuring apparatus, and post-selecting at a later time. To have an intuition about weak measurement on pre- and postselected ensembles, take the 3-box problem [5]. Here, at time $t=0$ a state $|\psi\rangle$ is prepared in a superposition of states $|A\rangle,|B\rangle$ and $|C\rangle$ (the three boxes), e.g. $|\psi\rangle=\frac{1}{\sqrt{3}}(|A\rangle+|B\rangle+|C\rangle)$. At a later time $t=1$ the system is weakly measured in the basis $\{|A\rangle,|B\rangle,|C\rangle\}$ and then post-selected in some other state $|\phi\rangle$ which is not orthogonal to $|\psi\rangle$, e.g. $|\phi\rangle=\frac{1}{\sqrt{3}}(|A\rangle+|B\rangle-|C\rangle)$. The resulting weak values of the operator projecting into the three boxes at time $t=1$ with post-selection in state $|\phi\rangle$ at time $t=2$, calculated from Eq. (2), are, respectively,

$$
A_{w}=1, \quad B_{w}=1, \quad C_{w}=-1 .
$$

\footnotetext{
* sina.salek@bristol.ac.uk †roman.schubert@bristol.ac.uk †.wiesner@bristol.ac.uk
}

These results can also be mathematically studied in the framework of Dirac quasi-probabilities. In 1945, Paul Dirac introduced a complex phase-space distribution to make an "Analogy Between Classical and Quantum Mechanics" [6], given by

$$
\operatorname{Pr}\left(a_{m}, b_{n}\right)=\operatorname{Tr}\left[\rho \Pi_{A}^{m} \Pi_{B}^{n}\right],
$$

where the $a_{m}$ and $b_{n}$ are the eigenvalues of the operators $\Pi_{A}$ and $\Pi_{B}$, and $\Pi_{A}^{m}$ and $\Pi_{B}^{n}$ are the projectors onto the corresponding eigenstates.

The Dirac distribution (44) satisfies all the conditions of classical Kolmogorov probabilities, except that it is not a positive real function. It was shown that the negativity and complexness of this function is due to the non-commutativity of the quantum mechanical observables [7]. The Dirac distribution is normalised and gives correct marginals,

$$
\sum_{n} \operatorname{Pr}\left(a_{m}, b_{n}\right)=\sum_{n} \operatorname{Tr}\left[\rho A_{m} B_{n}\right]=\operatorname{Tr}\left[\rho A_{m}\right]
$$

and

$$
\sum_{m} \operatorname{Pr}\left(a_{m}, b_{n}\right)=\sum_{m} \operatorname{Tr}\left[\rho A_{m} B_{n}\right]=\operatorname{Tr}\left[\rho B_{n}\right],
$$

it obeys the sum rule and the product rule, and it is compatible with Bayes' law.

Note, that Dirac distributions are not limited to phase space. In fact any two operators with non-vanishing overlap between each of their eigenstates can be used to construct a Dirac decomposition,

$$
\rho=\sum_{m, n} \operatorname{Pr}\left(a_{m}, b_{n}\right) \frac{\left|a_{m}\right\rangle\left\langle b_{n}\right|}{\left\langle b_{n} \mid a_{m}\right\rangle},
$$

as long as the operators have the same Hilbert space dimension as the state $\rho$, and their eigenvectors are mutually non-orthogonal and none of those eigenvectors are orthogonal to the state $\rho$. Hence a space spanned by any two such observables would be sufficient to describe all the information available from the state $\rho$. This is due to the fact that one can describe any quantum state of $d$-dimensional Hilbert Space with $d^{2}-1$ elements. 
For theoretical considerations on the Dirac distribution see the work by Johansen [7] and Hofmann [8]. An experimental procedure for measuring the Dirac distribution of a general quantum state has been given by Lundeen and Bamber [9].

We will now describe how weak values can be understood in the framework of Dirac distributions.

Note that we can rewrite the weak value, Eq. (2), as

$$
\Pi_{w}=\frac{\langle\phi|\Pi| \psi\rangle}{\langle\phi \mid \psi\rangle} .
$$

Now we can interpret $\Pi_{w}$ as a conditional Dirac distribution of an ensemble which is pre- and post-selected in states $|\psi\rangle$ and $|\phi\rangle$, respectively. Using Bayes' law and defining $\rho_{\psi}:=|\Psi\rangle\langle\Psi|$ and $\rho_{\phi}:=|\Phi\rangle\langle\Phi|$, the weak value can be written as

$$
\Pi_{w}=\operatorname{Pr}(\Pi \mid \phi)=\frac{\operatorname{Tr}\left[\rho_{\psi} \rho_{\phi} \Pi\right]}{\operatorname{Tr}\left[\rho_{\psi} \rho_{\phi}\right]} .
$$

In this last equation the interpretation as a conditional quasi-probability follows from the use of Bayes' law.

This close connection between weak values and Dirac quasi-probabilities gives an operational meaning to the complex values of the Dirac distribution as a result of weak measurements. In the case where the measurement was performed by coupling the momentum of the measurement pointer to the quantum system, the real part of the weak value refers to the shift in the position of the measurement pointer, while the imaginary part refers to the shift in the momentum of the measurement pointer [10]. The same interpretation can be given to the real and complex part of the Dirac distribution. Given this operational meaning of the Dirac distribution, we proceed to use this formalism to make an analogy between classical conditional probabilities and quantum conditional states.

In the following, we establish first that Eq. (1) is actually a form of a conditional state by using the framework of Dirac distributions. This allows us then to define a corresponding conditional entropy of post-selected quantum states.

\section{QUANTUM CONDITIONAL STATES AND CONDITIONAL ENTROPY}

\section{A. Quantum states, conditioned on post-selection}

Using the conditional Dirac distribution obtained in Eq. (9), we now construct the corresponding conditional Dirac decomposition $\rho_{\psi \mid \phi}$ in analogy to Eq. (77), given post-selection in some state $|\phi\rangle$.

The summation here runs over the eigenstates of a projection operator $\Pi$ measured weakly in between the times of pre-selection and post-selection. We define $\Pi:=|h\rangle\langle h|$, where $|h\rangle$ is one out of a complete set of basis states $\{|h\rangle\}$ which are mutually non-orthogonal to the state $|\phi\rangle .[13]$
The conditional Dirac decomposition $\rho_{\psi \mid \phi}$ we obtain is

$$
\rho_{\psi \mid \phi}:=\sum_{\mathrm{h}} \operatorname{Pr}(\mathrm{h} \mid \phi) \frac{|\mathrm{h}\rangle\langle\phi|}{\langle\phi \mid \mathrm{h}\rangle} .
$$

$\rho_{\psi \mid \phi}$ is the density operator of all paths leading from state $|\psi\rangle$ to post-selected state $|\phi\rangle$. One may note that the operator $\rho_{\psi \mid \phi}$ is not Hermitian. It has been argued in the weak measurement literature why this should not be a cause of concern [9]. $\rho_{\psi \mid \phi}$ thus defined is a trace-one operator and can indeed, by construction, be determined by weak measurements. In the case of the three-box problem, the eigenstates $|h\rangle$ of the projector $\Pi$ would be $|A\rangle$, $|B\rangle$, and $|C\rangle$, representing the system in being in one of the three boxes $A, B$, and $C$ at time $t=1$.

By writing

$$
\operatorname{Pr}(\mathrm{h} \mid \phi)=\frac{\langle\phi \mid \mathrm{h}\rangle\langle\mathrm{h} \mid \psi\rangle}{\langle\phi \mid \psi\rangle}
$$

and inserting it into Eq. (10) we obtain

$$
\begin{aligned}
\rho_{\psi \mid \phi} & =\sum_{\mathrm{h}} \frac{|\mathrm{h}\rangle\langle\mathrm{h} \mid \psi\rangle\langle\phi|}{\langle\phi \mid \psi\rangle} \\
& =\frac{|\psi\rangle\langle\phi|}{\langle\phi \mid \psi\rangle},
\end{aligned}
$$

which is indeed the generalised density operator for postselected ensembles, defined in Eq. (1). This links the interpretation Eq. (10) as conditional quantum states to the generalised density operator for post-selected ensembles as in Eq. (11).

We observe that the application of Bayes' law in the definition (10) results in a density operator which is the extension of classical conditional probabilities. This analogy can be further clarified by multiplying $\rho_{\psi \mid \phi}$ by probability $\operatorname{Pr}(\phi)$ of the system ending in state $|\phi\rangle$ and sum Eq. (10) over a complete basis $\{|\phi\rangle\}$. Thus, we retrieve the density operator of Eq. (7) which contains the full information about the system,

$$
\rho=\sum_{\phi} \operatorname{Pr}(\phi) \rho_{\psi \mid \phi},
$$

with $\operatorname{Pr}(\phi)=\operatorname{Tr}[\rho|\phi\rangle\langle\phi|]$ being the probability of the state being in the state $|\phi\rangle$ at the time of post-selection. This is what is expected from the analogy with classical probabilities.

\section{B. Entropies, conditioned on post-selection}

In classical information theory, the entropy of random variable $X$ conditioned on selection of a particular instance $y$ of random variable $Y$ is $H(X \mid Y=y)=$ $-\sum_{x} \operatorname{Pr}(x \mid y) \log \operatorname{Pr}(x \mid y)$, where $\operatorname{Pr}(x \mid y)$ is the conditional probability of $x$ given the occurrence of $y$. Note that this function is the average $\langle-\log \operatorname{Pr}(x \mid y)\rangle$. The 
classical conditional entropy is, again, an average, i.e. $H(X \mid Y)=\sum_{y} \operatorname{Pr}(y) H(X \mid Y=y)$.

In analogy to the classical conditional entropy, we define the conditional entropy of a state $|\psi\rangle$, given postselection in one out of a possible set of states $|\phi\rangle$, to be

$$
S_{c}(\psi \mid \Phi=\phi)=-\frac{1}{2} \operatorname{Tr}\left[\rho_{\psi \mid \phi} \log \left(\rho_{\psi \mid \phi} \rho_{\psi \mid \phi}^{\dagger}\right)\right],
$$

where $\rho_{\psi \mid \phi}^{\dagger}$ is the conjugate transpose of $\rho_{\psi \mid \phi}$. We note that this particular form of the entropy is an average of the logarithm of the density operator of the system:

$$
S_{c}(\psi \mid \Phi=\phi)=\left\langle-\log \left(\left(\rho_{\psi \mid \phi} \rho_{\psi \mid \phi}^{\dagger}\right)^{1 / 2}\right)\right\rangle .
$$

One could choose other functional forms for the conditional entropy here. For instance, one could choose the function $-\frac{1}{2} \operatorname{Tr}\left[\rho_{\psi \mid \phi} \rho_{\psi \mid \phi}^{\dagger} \log \left(\rho_{\psi \mid \phi} \rho_{\psi \mid \phi}^{\dagger}\right)\right]$ instead. However, this would not correspond to an average of the logarithm. This makes our choice for the conditional entropy function a natural one. Note that the argument of the logarithm gives the Singular values of the operator $\rho_{\psi \mid \phi}$. The Singular Value Decomposition is a generalisation of diagonalisation needed here due to the particular choice of basis in the definition of the two-state density operator (Eq. 10). For Hermitian operators, such as ordinary density operators, the singular values and the eigenvalues are the same.

Finally, we define the general quantum conditional entropy as an average of the entropy conditioned on a particular choice of post-selection as

$$
S_{C}(\psi \mid \Phi)=\sum_{\phi} \operatorname{Pr}(\phi) S_{c}(\psi \mid \Phi=\phi) .
$$

Eqs. (14-16) can be simplified. By inserting Eq. (1) into Eq. (14) we obtain

$$
\begin{aligned}
S_{c}(\psi \mid \Phi=\phi) & =-\frac{1}{2} \frac{\left\langle\phi\left|\log \left(\rho_{\psi \mid \phi} \rho_{\psi \mid \phi}^{\dagger}\right)\right| \psi\right\rangle}{\langle\phi \mid \psi\rangle} \\
& =\log |\langle\phi \mid \psi\rangle| .
\end{aligned}
$$

Inserting this into Eq. (16) we obtain the simplified expression

$$
S_{C}(\psi \mid \Phi)=\sum_{\phi}|\langle\phi \mid \psi\rangle|^{2} \log |\langle\phi \mid \psi\rangle| .
$$

This new expression is very useful for finding upper and lower bounds of the conditional entropy. We can see that $S_{C}$ has a negative lower bound of $\frac{1}{d} \log \frac{1}{\sqrt{d}}$, where $d$ is the dimension of the Hilbert Space. This lower bound is reached when all states $|\phi\rangle$ have the same magnitude in overlap with the state $|\psi\rangle$. $S_{C}$ is bounded from above by the von Neumann entropy of the system without postselection.

The conditional entropy $S_{C}$ can be understood as the amount of information contained in a system conditioned on a particular post-selection. The case discussed here is the special case of pre-selection in a pure state which, by definition, has zero von Neumann entropy. The upper bound of the conditional entropy is zero in this case and thus the conditional entropy of a pure pre-selected state $|\psi\rangle$ can be negative. This is a necessary consequence from choosing a subset of the entire (pure) system for post-selection which necessarily decreases the entropy below zero. Thus, a negative conditional entropy of postselected ensembles becomes intuitive as a subset contains less information than the whole. The concept of negative conditional entropy appears in other contexts in quantum information. For instance, for a bipartite system $\rho_{A B}$ in a pure, i.e. maximally entangled state, the joint entropy is zero. On the other hand, the locally the subsystems $\rho_{A}$ and $\rho_{B}$ are in maximally mixed states. Hence, the entropy of one subsystem, conditioned on the measurement of the other, $S\left(\rho_{A} \mid \rho_{B}\right)=S\left(\rho_{A B}\right)-S\left(\rho_{B}\right)<0$, takes on a negative value. This conditional entropy was also calculated in the form of $S\left(\rho_{A} \mid \rho_{B}\right)=-\operatorname{Tr}\left[\rho_{A B} \log \rho_{A \mid B}\right]$, where $\rho_{A \mid B}=\lim _{n \rightarrow+\infty}\left[\rho_{A B}^{1 / n}\left(\mathbb{1}_{A} \otimes \rho_{B}\right)^{-1 / n}\right]^{n}[11]$ which is closer in outlook to the construction introduced in Eq. (16).

a. Example: 3-box post-selection We will now revisit the 3-box problem [5] from the beginning and calculate the conditional entropies just defined.

Choosing pre- and post-selection as before, i.e. $|\psi\rangle=$ $1 / \sqrt{3}(|A\rangle+|B\rangle+|C\rangle)$ and $|\phi\rangle=1 / \sqrt{3}(|A\rangle+|B\rangle-|C\rangle)$, one obtains for the conditional entropy $S_{c}$ of the system, Eq. (14), $S_{c}(\psi \mid \Phi=\phi)=-\ln 3$. In order to calculate the conditional entropy $S_{C}$, Eq. (16), given that the Hilbert space dimension of the system is three, one needs to perform the same calculation as above for two other postselected states, $\left|\phi^{\prime}\right\rangle$ and $\left|\phi^{\prime \prime}\right\rangle$. The only restrictions on the choice of these two post-selected states are that they need to be mutually non-orthogonal to the state of each box, i.e. to $|A\rangle,|B\rangle$ and $|C\rangle$. And in addition they need, together with our original state $|\phi\rangle$, to span the Hilbert space of the state $|\psi\rangle$. Take these states to be

$$
\left|\phi^{\prime}\right\rangle=\frac{1}{\sqrt{3}}|A\rangle+\frac{-3-\sqrt{3}}{6}|B\rangle+\frac{-3+\sqrt{3}}{6}|C\rangle
$$

and

$$
\left|\phi^{\prime \prime}\right\rangle=\frac{1}{\sqrt{3}}|A\rangle+\frac{3-\sqrt{3}}{6}|B\rangle+\frac{3+\sqrt{3}}{6}|C\rangle .
$$

Calculating $S_{c}$ of Eq. (14) for for $\left|\phi^{\prime}\right\rangle$ and $\left|\phi^{\prime \prime}\right\rangle$ gives $S_{c}\left(\psi \mid \Phi=\phi^{\prime}\right)=-\log _{3} 4.10$ and $S_{c}(\psi \mid \Phi=\phi)=$ $-\log _{3}$ 1.10. With probabilities $\operatorname{Pr}(\phi)=0.11, \operatorname{Pr}\left(\phi^{\prime}\right)=$ 0.06 and $\operatorname{Pr}\left(\phi^{\prime \prime}\right)=0.83$ (given by $|\langle\phi \mid \psi\rangle|^{2}$ and correspondingly for $\phi^{\prime}$ and $\left.\phi^{\prime \prime}\right)$, the conditional entropy Eq. (16) becomes $S_{C}(\psi \mid \Phi)=-0.26$, with the logarithm being calculated in base 3 for convenience.

The 3-box problem illustrates the negativity of the conditional entropy where each path through a box contains partial information of the system as a whole.

b. Conclusion In this work we have defined an entropy for post-selected ensembles. To this end, we 
have adapted the formalism of Dirac quasi-probabilities, and showed that the two-state density operator of postselected ensembles is a generalisation of the classical conditional probability, given that a particular final state is selected. We have found upper and lower bounds on such entropies and interpreted the values as the amount of information contained in a system conditioned on a particular post-selection. Furthermore, we showed that these states have properties beyond their classical counterparts. Most notably, conditional quantum entropies as defined in this work can have negative values. This is consistent with our interpretation. This new quantum entropy should open up avenues for studying the properties of weak measurement of more general states than discussed in this paper, such as mixed post-selected states.

Acknowledgements We thank Jeff Lundeen, Holger Hofmann and Sandu Popescu for their helpful comments and stimulating discussions. K.W. thanks EPSRC for financial support. $\begin{array}{lccccc}\text { [1] B. } & \text { Reznik } & \text { and } & \text { Y. } & \text { Aharonov, } & \text { Phys. } \\ \text { Rev. } & \text { A } & \mathbf{5 2} & 2538 & (1995) & \text { URL }\end{array}$ http://link.aps.org/doi/10.1103/PhysRevA.52.2538

[2] A. Hosoya and Y. Shikano, Journal of Physics A: Mathematical and Theoretical 43, 385307 (2010).

[3] R. Silva, Y. Guryanova, N. Brunner, N. Linden, A. J. Short, and S. Popescu, Physical Review A 89, 012121 (2014).

[4] Y. Aharonov, D. Z. Albert, and L. Vaidman, Phys. Rev. Lett. 60, 1351 (1988), URL http://link.aps.org/doi/10.1103/PhysRevLett.60.1351

[5] Y. Aharonov and L. Vaidman, Journal of Physics A: Mathematical and General 24, 2315 (1999).

[6] P. A. M. Dirac, Rev. Mod. Phys. 17, 195 (1945), URL http://link.aps.org/doi/10.1103/RevModPhys.17.195,

[7] L. M. Johansen, Phys. Rev. A 76, 012119 (2007), URL
http://link.aps.org/doi/10.1103/PhysRevA.76.012119

[8] H. F. Hofmann, New J. Phys. 14, 043031 (2012).

[9] J. S. Lundeen and C. Bamber, Phys. Rev. Lett. 108, $070402 \quad$ (2012), URL http://link.aps.org/doi/10.1103/PhysRevLett.108.070402

[10] R. Jozsa, Phys. Rev. A 76, 044103 (2007), URL http://link.aps.org/doi/10.1103/PhysRevA.76.044103

[11] N. J. Cerf and C. Adami, Phys. Rev. Lett. $\mathbf{7 9 ,} 5194 \quad$ (1997), URL http://link.aps.org/doi/10.1103/PhysRevLett.79.5194

[12] J. B. Hartle, Physical Review A 78, 012108 (2008).

[13] We use the letter $h$ for the basis states of the projection operator to honour the closeness of these concepts to the consistent histories approach of quantum measurement, see [12]. 